\title{
Anorexia nervosa con desenlace fatal: propósito de un caso.
}

\author{
Anorexia nervosa with fatal outcome: A case report. \\ Stephanie Aliaga-Tinoco ${ }^{1,2, a}$, Lizardo Cruzado ${ }^{1,2, b}$.

\section{RESUMEN}

La Anorexia Nervosa (AN) es el trastorno psiquiátrico con mayor nivel de mortalidad directa y, a la vez, causa de múltiples y riesgosas complicaciones somáticas tales como: bradicardia, hipotensión ortostática, arritmias cardiacas, hipoglicemia, falla hepática, alteraciones hidroelectrolíticas y hormonales, osteopenia, dilatación gástrica y, finalmente, muerte súbita por diferentes causas. A pesar de su prevalencia relativamente baja, afecta a personas jóvenes con larga expectativa de vida. Su manejo, cuando llega a niveles de gravedad severa (IMC < 15), es complejo, pues requiere trabajo conjunto de psiquiatría y otras especialidades médicas y puede tener consecuencias ominosas. Se presenta y discute el caso de una paciente mujer de 19 años cuyo IMC llegó a 11 y que, lamentablemente, falleció mientras recibía atención hospitalaria. Se revisan las principales complicaciones médicas de la AN, remarcándose la necesidad de considerar los parámetros pertinentes para una atención hospitalaria oportuna que pueda evitar desenlaces fatales.

PALABRAS CLAVE: Anorexia nerviosa; Desnutrición Proteico-Calórica; Falla hepática; Muerte súbita.

\section{SUMMARY}

Anorexia Nervosa (AN) is the psychiatric disorder with the highest direct mortality level and, at the same time, the cause of multiple and risky somatic complications such as bradycardia, orthostatic hypotension, cardiac arrhythmias, hypoglycemia, liver failure, hydro-electrolytic and hormonal disorders, osteopenia, gastric dilation and, finally, sudden death from different causes. Despite its relatively low prevalence, it affects young people with a long life expectancy. Its medical management, when reaching a high severity level $(\mathrm{BMI}<15)$, is complex as it requires joint work between psychiatry and other medical specialties, and can even have ominous consequences. The case of a 19-year-old woman whose BMI was around 11, and who unfortunately died while receiving hospital care, is discussed. The main complications of AN are reviewed, and the need to consider the relevant parameters for a timely hospital care are highlighted, in order to avoid fatal outcomes.

KEYWORDS: Anorexia nervosa; protein-energy malnutrition; liver failure; sudden death.

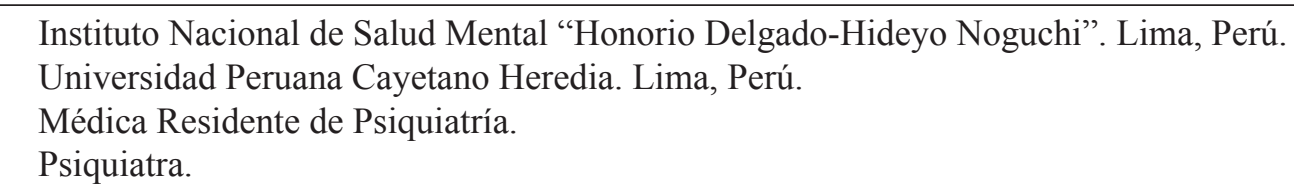




\section{INTRODUCCIÓN}

La anorexia nervosa (AN) ostenta la mayor tasa de mortalidad de todos los trastornos psiquiátricos. El 90\% de quienes padecen AN son mujeres: una mujer joven y anoréxica tiene hasta 10 a 12 veces más riesgo de morir que una mujer saludable (1). Definida en la CIE-10 como la pérdida de peso intencional a partir de la idea sobrevalorada de temor a la obesidad y flacidez corporal, la AN conduce a desnutrición y alteraciones corporales diversas que pueden acarrear graves complicaciones. En nuestro medio, se ha detectado entre adolescentes una tasa de obsesión por la delgadez de $3,4 \%$ y prevalencia de conductas restrictivas de $1,6 \%$; en este grupo etario, la prevalencia de $\mathrm{AN}$ es $0,2 \%$ de la población femenina (2). Se calcula que la prevalencia de vida de AN entre adolescentes y adultos jóvenes es $0,5 \%$ (3); aunque usando criterios más abarcativos, esta puede llegar a más de $2 \%$ (4). Si bien la AN predomina en adolescentes, el inicio de esta patología a partir de la edad adulta es indicativo de peor pronóstico (5).

La AN tiene evolución variable: si bien la mitad de pacientes supera totalmente el padecimiento, un $30 \%$ presenta mejoría parcial (muchas pacientes pasan a desarrollar bulimia), un $20 \%$ presenta síntomas de la enfermedad crónica y hasta 5\% puede llegar a fallecer como producto de la misma (5). A diferencia de otros trastornos psiquiátricos, los trastornos de la conducta alimentaria (TCA) y especialmente la AN, requieren atención médica concurrente por la serie de comorbilidades que se presentan en diferentes momentos de su evolución, sobre todo a medida que el índice de masa corporal se reduce peligrosamente (6). Entre las comorbilidades somáticas de la AN deben mencionarse, como las más frecuentes: las cardiovasculares, sobre todo la bradicardia e hipotensión; las de tipo hepático, con falla hepática, así como las hidroelectrolíticas, en caso de vómitos reiterados, con posible repercusión en arritmias cardiacas potencialmente fatales (6). De hecho, entre un tercio y la mitad de los fallecimientos en AN son por muerte súbita cardiaca (7). Se ha descrito de manera constante una tasa de mortalidad de 1 de cada 20 pacientes con AN (5). Aparte de las comorbilidades asociadas a la malnutrición severa, debe señalarse que una causa importante de mortalidad es el suicidio: hasta $20 \%$ de pacientes con AN lo intentan y una de cada 5 muertes en estas pacientes es por suicidio -más frecuente en la AN purgativa que en AN restrictiva(8). En comparación, las pacientes con bulimia nervosa no registran un aumento de mortalidad prematura, y si bien intentan el suicidio en similar proporción a los pacientes con AN, lo consolidan en mucha menor cuantía debido al uso de medios menos letales. (7)

A propósito de un caso de $\mathrm{AN}$ con desenlace fatal, presentamos una revisión de la literatura, con énfasis en los principales problemas clínicos que acarrean riesgo letal y los criterios de severidad de estas complicaciones a fin de que los pacientes afectados puedan oportunamente recibir la ayuda médica especializada correspondiente.

\section{Caso clínico}

Mujer de 20 años de edad, nacida en el año 1995, soltera, que cursaba estudios universitarios, vivía con padres y un hermano cinco años menor. Desde los 12 años, cuando pesaba $56 \mathrm{~kg}$, la fastidiaban por ser "llenita", lo que la incomodaba y la motivaba a seleccionar algunos alimentos, pero sin mayor repercusión clínica. A los 19 años pesaba $58 \mathrm{~kg}$, y su talla era de 1,59 (índice de masa corporal, IMC: 22,7). Refiere que a esa edad (fines del año 2014), ante el estrés de los estudios universitarios, si ingería algún alimento adicional (golosina o postre), se preocupaba por el exceso de calorías y esta inquietud fue progresivamente en aumento, al punto de llegar a calcular las calorías de todo lo que ingería y sentir ansiedad si sobrepasaba la ingesta diaria adecuada para ella; ella se veía a sí misma como "llenita". Para compensar esto, decidió además hacer diariamente ejercicio físico intenso. Al cabo de seis meses fue llevada a un centro de salud donde se verificó pérdida ponderal de $15 \mathrm{~kg}$ (IMC: 17), se le diagnosticó "gastritis emotiva" y una psicóloga sugirió que padecía anorexia y debía ser evaluada por psiquiatría. Refirió además fatigabilidad y dificultad para concentrarse, por lo que abandonó sus estudios universitarios.

Luego de un año y un mes desde el inicio de sus síntomas, tuvo su primera consulta psiquiátrica, para ese entonces reportó amenorrea de cuatro meses, restricción dietética y ejecución de ejercicio físico intenso pero sin empleo de diuréticos, laxantes ni provocación de vómitos. Refería que si comía una fruta, por ejemplo, consideraba esto como una comida completa y debía esperar cuatro horas para ingerir otro alimento, además de que estaba muy preocupada por el peso y sus implicancias en la salud. Había llegado a pesar $35 \mathrm{~kg}$ (IMC: 13,8). Se le diagnosticó anorexia nervosa de tipo restrictivo, se le prescribió fluoxetina $20 \mathrm{mg}$ diarios y psicoterapia. 
Durante las siguientes consultas mensuales no se registró mayor variación del peso. Se anota que la paciente mantenía ansiedad ante la ingesta de alimentos por la probabilidad de subir de peso, verificaba las calorías y había discusiones frecuentes a la hora de las comidas. Recién un año y dos meses después del inicio de síntomas fue evaluada por consulta de medicina interna. Dos meses luego accedió a consultorio de Trastornos de Consulta Alimentaria (TCA) de un hospital psiquiátrico especializado, donde se verificó peso de 27,4 kg (IMC: 10,8), se cambió fluoxetina por sertralina $50 \mathrm{mg}$. diarios y como además presentaba dolor abdominal difuso, estreñimiento, y en el hemograma mostraba 3400 leucocitos por mililitro, se solicitó interconsultas con otras especialidades médicas. La familia de la paciente no secundó plenamente la terapia y la adherencia distó de ser completa.

Mediante consultas semanales en el consultorio de TCA, énfasis en la restricción de ejercicio físico y vigilancia de su ingesta a lo largo de tres meses que permaneció en este programa, se logró recuperar su peso hasta 29,8 $\mathrm{kg}$ (IMC 11,8), en ese entonces registraba presión arterial (PA): $80 / 50 \mathrm{~mm}$ HG y frecuencia cardiaca (FC): $68 \mathrm{x}^{\prime}$. Lamentablemente, luego de un mes (11/05/2016), acudió por emergencia de hospital general por extrema debilidad: los días previos solo había ingerido uno o dos vasos de leche de soya al día. Esa misma mañana fue referida desde el hospital general, sin más examen, al hospital psiquiátrico de origen, donde se la encontró despierta, hipoactiva, escueta, asténica, con ánimo decaído, refería verse adelgazada, tenía edemas en los pies e intolerancia al frío. Su (PA) era 80/50 mm Hg, (FC) de 50 x', pesaba $27 \mathrm{~kg}$ (IMC: 11,1). Quedó en observación en la emergencia del hospital psiquiátrico; se implementó hidratación parenteral y dieta enteral de $1000 \mathrm{kcal}$ fraccionada. Tenía hemoglobina $10,5 \mathrm{~g} / \mathrm{dL}$; potasio sérico: $3,24 \mathrm{mEq} / \mathrm{L}$, y a lo largo de las primeras horas se registró bradicardia sinusal $(35 \mathrm{x}$ ').

Al mediodía se insistió en referirla nuevamente a la emergencia del hospital general, donde permaneció por aproximadamente 6 horas, se le proporcionó hidratación parenteral y al anochecer fue enviada de vuelta al hospital psiquiátrico (se dijo a la madre que la paciente se hallaba "estable" y debía recibir "atención psiquiátrica"). Nuevamente aquí se verificó PA: 80/50 mm Hg, FC: 56 x', la paciente se quejaba de hallarse fatigada y débil, y quedó con una vía endovenosa de $\mathrm{NaCl} 0,9 \%$ a XV gotas $\mathrm{X}$ '. Súbitamente a las 7 de la mañana del siguiente día, la paciente fue hallada con dificultad respiratoria y no respondía al llamado (solo 10 minutos antes había pedido desde su cama que le hagan la higiene diaria). Presentaba PA: 80/50 mm Hg, FC: $66 x^{\prime}$, frecuencia respiratoria (FR): 28 $\mathrm{x}^{\prime}$, con saturación de $93 \%$, periodos de apnea, leve respuesta motora al dolor, apertura ocular apenas perceptible, Glasgow 4/10, por lo que se decidió referir inmediatamente a emergencia de hospital general.

Ingresó a unidad de Trauma-shock a las 8 a.m. con PA: 103/50 mm Hg, FC: $115 x^{\prime}$, FR: 15 x', T: 36, $8^{\circ}$ C, saturación $\mathrm{O}_{2}: 98 \%$. Su glucemia fue $26 \mathrm{mg} / \mathrm{dL}$. Se le transfundió $500 \mathrm{ml}$ de $\mathrm{NaCl} 0,9 \%$ y 5 ampollas de dextrosa al $33 \%$, además de dextrosa $5 \%$ con 2 ampollas de $\mathrm{NaCl}$ al $20 \%$ a $90 \mathrm{ml}$ por hora. También se halló hipokalemia: $2,47 \mathrm{mEq} / \mathrm{mL}$, elevación marcada de transaminasas: TGO: $1633 \mathrm{U} / \mathrm{L}$, TGP: 1324, hemoglobina $11,3 \mathrm{mg} / \mathrm{dL}$, leucocitos 14680 $\mathrm{mL}$, plaquetas $163000 \mathrm{~mL}$, su nivel de conciencia fue mejorando y se mantenía hemodinámicamente estable, sin soporte ventilatorio, se instalaron retos de potasio para normalizar sus niveles séricos. El INR era 3,07.

A la medianoche de ese mismo día (12.05.2016) presentaba PA: 100/60 mm Hg, FC: 82x', FR: 20x' y T: $36^{\circ} \mathrm{C}$, recibía alimentación por sonda nasogástrica $800 \mathrm{~mL}$ en 4 tomas, vitaminoterapia (complejo B) y $\mathrm{KCl}$ al 20\% una ampolla en $1 \mathrm{~L}$ de dextrosa $5 \%$ cada 12 horas. No había cambios electrocardiográficos y se observaron algunas equimosis en la piel.

Al mediodía del siguiente día (13/05) mantenía hipoglicemia sintomática $(45 \mathrm{mg} / \mathrm{dL})$ y se mostraba somnolienta. Su PA era 80/60, FC 75 x'. Dos horas después su PA era 70/50, FC: 96 x', saturación $\mathrm{O}_{2}$ $90 \%$ con $\mathrm{FiO}_{2} 21 \%$, Glasgow 14/15, Bilirrubina total: $1,2 \mathrm{mg} / \mathrm{dl}$, Bilirrubina indirecta: $0,9 \mathrm{mg} /$ dl, Na: $133 \mathrm{mEq} / \mathrm{L}, \mathrm{K}: 4,3 \mathrm{mEq} / \mathrm{L}, \mathrm{Cl}: 96 \mathrm{mEq} / \mathrm{L}$, Fósforo: 0,9 mg/mL, GGT: 188, DHL: 2017. El INR había ascendido a 5,26. Se planteó diagnóstico de compromiso hepatocelular (transaminasas elevadas + INR prolongado), síndrome de realimentación (IMC: 11, hipofosfatemia severa, episodios de hipoglucemia e hipokalemia), en el contexto de la anorexia severa y desnutrición. Se replantea la dieta: proteínas: $1 \mathrm{gr} /$ $\mathrm{kg} /$ día, calorías: $20 \mathrm{kcal} / \mathrm{kg} / \mathrm{día}, \mathrm{Na}^{+}$menos de $2 \mathrm{gr} /$ día, VT: $1000 \mathrm{cal} /$ día, monitoreo estricto de sodio, potasio, cloro, magnesio, fosforo y calcio, vitamina $\mathrm{K}$ 1 amp IV diaria por 3 días.

A las 8 pm se registró PA: 76/48 mm Hg, FC: 95 x', FR: 20, Sat $\mathrm{O}_{2}: 97 \%$, diuresis: $800 \mathrm{~mL}$, balance hídrico: $+13,4$; residuo gástrico: $20 \mathrm{~mL}$. Se administró potasio, fósforo y gluconato de calcio, plasma fresco 
congelado, vitaminas $\mathrm{K}$ y complejo $\mathrm{B}$, omeprazol y metoclopramida. Se registró murmullo vesicular abolido en la base del hemitórax derecho que luego se extiende a todo el hemitórax derecho. La paciente se quejaba de falta de aire. Sat $\mathrm{O}_{2}: 96 \%$ con $\mathrm{FiO}_{2}: 50 \%$. PA: $80 / 50 \mathrm{~mm} \mathrm{Hg}, \mathrm{FC}: 100 \mathrm{x}^{\prime}$. En radiografía se nota neumotórax derecho completo, sin desplazamiento de mediastino. Se trata de mejorar el INR antes de la colocación de tubo torácico.

Al día siguiente $(14 / 05)$ se apreció discreta reexpansión de pulmón derecho. La paciente se mantenía despierta pero se quejaba de sensación de ahogamiento por recibir alimentos por vía oral teniendo colocada la sonda nasogástrica. Se solicitó nuevamente panel de exámenes auxiliares. Registró PA: 92/70 mm Hg, FC: 68 x', FR: 18 x', Sat $\mathrm{O}_{2}: 96 \%$. Hemodinámicamente estable, pasa de Trauma-shock a Observación al anochecer. La paciente lucía en mal estado general, con palidez, ictericia y equimosis a predominio de miembros inferiores. Se evidenciaba en el hemitórax derecho murmullo vesicular abolido, abdomen excavado. Cerca de medianoche súbitamente presentó paro cardiaco. Se le aplicó amiodarona, epinefrina y atropina y se la desfibriló en tres oportunidades sin respuesta positiva. Tras 30 minutos de reanimación, se la declaró fallecida.

\section{DISCUSIÓN}

Estamos ante el desenlace fatal de un caso grave de anorexia nervosa, de tipo restrictivo. Aunque los autores de este reporte no fueron los médicos tratantes se puede evidenciar, ante el progresivo deterioro de salud de la paciente, cómo se intentó intervenir en su grave estado pero la inexistencia de un sistema integral que combine el manejo médico con la atención psiquiátrica dificultó la adecuada asistencia. Esto se demuestra en las recurrentes referencias de la paciente desde el hospital general al hospital psiquiátrico, y viceversa, hasta el desenlace final. Pero aún desde bastante antes se aprecia que, pese a la disminución grave de su IMC, no se consiguió su internamiento. Evaluando retrospectivamente el caso, es evidente que la cadena de sucesos que llevó al desenlace final, pudo iniciarse en varios momentos previos. Esto lamentablemente sucede incluso en sistemas de salud de países del primer mundo. En Inglaterra hace poco tiempo se configuró la iniciativa MARSIPAN (Management of really sick patients with anorexia nervosa) (9) pues igualmente la integración de servicios médicos y psiquiátricos ante estos casos es bastante ardua: pero no solo en aspectos logísticos sino en los sustentos conceptuales, como si la división cartesiana mente-cuerpo prevaleciera ominosamente. Bibliografía previa de nuestro país recomendaba el internamiento por dos meses a pacientes con AN: de esa época a hoy, lamentablemente la capacidad de provisión de servicios ha decrecido (10).

Brevemente, la fisiopatología de la AN puede dividirse en aquella dependiente del ayuno y la emaciación, aquella dependiente de la purga (en caso de AN purgativa), y el síndrome de realimentación (6). Hallazgos accesorios pueden ser la intoxicación hídrica en algunos casos puntuales. Estructuramos nuestra discusión en cuatro puntos principales dependientes ellos de la emaciación y ayuno-: hipoglucemia, neumotórax, falla hepática y muerte súbita cardiaca; que fueron las complicaciones sucesivamente presentadas en el caso reportado. Finalmente analizaremos sucintamente el panorama general del cuadro (síndrome de realimentación) y los criterios que abonan a un manejo intrahospitalario de cuadros de AN.

A) Hipoglucemia: Es definida como nivel sérico de glucosa menor a $60 \mathrm{mg} / \mathrm{dL}$. Si una persona con AN experimenta confusión o mareo en ayunas, o también luego de 30 a 90 minutos después de las comidas, frecuentemente esto es debido a la hipoglucemia. Asimismo, el desarrollo de convulsiones y pérdida de conciencia, deben hacer sospechar el desarrollo de esta complicación que, en casos extremos puede conllevar daño cerebral y muerte(11).Enlamayoríadecasos, afortunadamente la hipoglucemia es leve y usualmente asintomática: en estos casos se desaconseja una monitorización exageradamente reiterativa o administración liberal de glucosa parenteral (los volúmenes de sobrecarga de líquido que puede manejar un corazón atrófico son bastante reducidos). El desarrollo de hipoglucemia sintomática empero, se asocia a un pobre pronóstico en AN y es potencialmente fatal -como finalmente aconteció con nuestra paciente(12). La depleción masiva de glucógeno hepático y la falla de los procesos de gluconeogénesis por carencia de sustratos suficientes son los mecanismos implicados (13). En casos con elevación importante de transaminasas como el nuestro, el riesgo de hipoglucemia se multiplica por cinco (13).

La manera adecuada de combatir esta complicación es restaurar las reservas de glucógeno hepático mediante la realimentación, y así permitir que los niveles de glucemia entre alimentos sean estables, 
pero mientras eso sucede la provisión prudente de glucosa intravenosa es obligatoria (14).

B) Neumotórax espontáneo: $\mathrm{Si}$ el neumomediastino espontáneo es un problema infrecuente (1 caso en 7000 a 12000 hospitalizaciones), el neumotórax espontáneo es aún más inusual (5 casos por 100000 internamientos). De hecho, hasta hace pocos años, no había casos reportados de neumotórax asociado a AN (15). Se ha demostrado en modelos animales que la malnutrición reduce la matriz proteica del parénquima pulmonar, incluyendo tejido conectivo y elástico, lo que conlleva a cambios de tipo enfisematoso, pero además disminuye la producción de surfactante, con incremento de la fricción pleural (16). En nuestro caso no hubo factores de riesgo para neumotórax como ejercicio extenuante previo, tabaquismo o consumo de marihuana, tos ni vómitos: el dolor torácico y la dificultad respiratoria fueron las manifestaciones cruciales para el diagnóstico aunque este problema no fue directamente la causa del deceso en nuestro caso. Aunque el curso del neumotórax espontáneo es benigno usualmente, en casos de AN se sugiere la hospitalización por las potenciales complicaciones. Se ha señalado la posibilidad de subdiagnóstico ante casos leves por la baja sospecha diagnóstica (15).

$\mathrm{Si}$ bien el neumotórax secundario a $\mathrm{AN}$ es infrecuente, y no hay casos reportados de mortalidad asociada - excepto un caso en la serie peruana de Albornoz (17)- hay otros cambios más comunes en el aparato respiratorio como debilidad de los músculos correspondientes incluyendo el diafragma, disminución de la capacidad aeróbica y pulmonar, que aumentan el riesgo de complicaciones como aspiración e infecciones, sobre todo en la contingencia del uso de sonda nasogástrica (12).

C) Falla hepática: No es usual que se alteren los niveles de proteínas séricas ni de la albúmina aún en casos avanzados de AN, lo que suele sorprender pues se asume a los valores de proteinemia como indicadores claros de desnutrición (11). Sin embargo, al ser la AN el prototipo de desnutrición primaria, no secundaria a causas inflamatorias o infecciosas, el mantenimiento de cifras dentro de márgenes normales de la albúmina, es lo esperable. Una de las alteraciones médicas más frecuentes en AN es la elevación de las enzimas transaminasas. Hasta hace poco tiempo, solía atribuirse este fenómeno al proceso de realimentación de las pacientes con $\mathrm{AN}$, que supuestamente excedía las capacidades metabólicas de un hígado exhausto. Sin embargo, hoy es claro que la hipertransaminasemia se suscita desde periodos previos a la realimentación (13). El incremento moderado $(<200 \mathrm{IU} / \mathrm{L})$ se observa hasta en el $75 \%$ de los pacientes con anorexia nerviosa, siendo menos común el mayúsculo incremento $>2000$ IU/L, que solo acontece cuando el IMC se acerca a 12 o es menor a esta cifra -en un inicio no se sospechó este problema en nuestro caso y se investigó hepatitis infecciosa-. Existe relación inversamente proporcional entre ambos valores: a menor nivel de IMC, mayor será la consiguiente elevación de transaminasas y este dato es un poderoso predictor de insuficiencia hepática como en nuestro caso, en que sucedió hipoglucemia catastrófica (18). Las alteraciones del International Normalized Ratio (INR) son asimismo más comunes en pacientes con elevaciones extremas de las transaminasas pero se asume que, dada la emaciación de tejido graso y la pobre absorción de lípidos, la exigua absorción de vitamina $\mathrm{K}$ juega un rol causal determinante. Los incrementos de bilirrubinas y fosfatasa alcalina, en cambio, son mucho menos comunes (19).

Aunque no se ha aclarado del todo el mecanismo fisiopatológico subyacente a la desmesurada elevación de transaminasas, actualmente el más aceptado es la autofagia inducida por la inanición. La autofagia es un proceso normal responsable de la degradación del exceso de proteínas citosólicas y organelas, mediante los lisosomas, con el objetivo de eliminar y eventualmente reciclar las macromoléculas resultantes. Tiene un importante papel en varios eventos biológicos tales como la remodelación celular durante el desarrollo y diferenciación, adaptación a condiciones de estrés y envejecimiento. La autofagia en AN tiene un rol dual: al inicio es protectora pues facilita el funcionamiento celular y biosíntesis a partir de las moléculas que se reciclan, pese a la escasez de nutrientes. Estudios de microscopía electrónica en AN con elevación extrema de transaminasas, es decir, cuadros avanzados, han demostrado una excesiva activación de la autofagia que conduce a lesión del hepatocito y muerte celular pero sin necrosis, además de la consiguiente falla hepática por mengua del parénquima hepático funcionante (19). Durante la muerte celular por autofagia se incrementa la permeabilidad de la membrana celular, lo que explica la salida masiva de transaminasas aún sin necrosis ni inflamación 
aparente (18).

Aún en la terapia de realimentación existe el riesgo de elevación importante de transaminasas pero, si se efectúa con la pauta dietética adecuada, este riesgo se contiene y las enzimas se van normalizando, lo que apuntala como factor etiológico de la hipertransaminasemia a la desnutrición. Los casos reportados de AN con falla hepática total y encefalopatía y coagulopatía son afortunadamente escasos, quizá porque la muerte sobreviene antes por otros mecanismos (13).

D) Alteraciones cardiovasculares y muerte súbita cardiaca: Hasta el $80 \%$ de pacientes con AN padecen complicaciones cardiacas, y el 30\% de muertes súbitas en pacientes con AN puede atribuirse a estos problemas, lo que hace a las complicaciones cardiovasculares las más temidas. Los cambios estructurales, alteraciones de la conducción y repolarización, cambios hemodinámicos, alteraciones vasculares periféricas y otras menos frecuentes, forman parte de las diversas anomalías de este sistema (20).

La bradicardia es el signo más frecuente (hasta $95 \%$ de pacientes con AN) y se atribuye a la hiperactivación del tono vagal, supuestamente destinada a la máxima preservación de la energía disponible por un organismo que sufre severa privación de nutrientes. Su elevada prevalencia debe apuntar incluso a la sospecha de AN en adolescentes que no revelan alteraciones de conducta alimentaria pero que presentan bradicardia (21). Por otro lado, la presencia de taquicardia en casos establecidos de AN debiera alertar sobre posibles cuadros infecciosos subyacentes. Concomitantemente, hay hipotensión sistólica y diastólica proporcional que se traduce en frecuentes episodios de ortostatismo y hasta de lipotimia y síncope. La disminución de masa miocárdica sin una concomitante variación en la dimensión de las válvulas cardiacas puede llevar a fenómenos de prolapso, frecuentemente (50\%) de la válvula mitral, y esto es causa de dolor precordial, pero muy raramente de insuficiencia cardiaca: aún con los grados de atrofia descritos, los pacientes con AN mantienen fracciones de eyección dentro de rangos normales (22) aunque el volumen minuto obviamente disminuye debido a la bradicardia (20).

Por mucho tiempo, se adjudicó a la prolongación del intervalo QT los casos de muerte súbita cardiaca en las pacientes anoréxicas, por su relación con arritmias malignas y fibrilación ventricular. Sin embargo en la actualidad tal hipótesis se ha revisado pues no hay diferencias sustanciales en el intervalo QT (aún en AN purgativa donde suele haber alteración hidroelectrolítica frecuente) sino más bien en la "dispersión" de este intervalo (distancia entre los intervalos QT más largo y más corto) y se considera al incremento de este factor como específicamente arritmogénico pues representa variabilidad marcada en la recuperación de la excitabilidad miocárdica (también puede entenderse como un índice de "irritabilidad" miocárdica). Igualmente se apunta como arritmogénica a la disminución de la variabilidad de la frecuencia cardiaca, descrita en la AN (12). Mas bien, ante prolongación evidente del intervalo QT debieran buscarse otras causas específicas como alteraciones electrolíticas o uso de fármacos cardiotóxicos (20).

Otras alteraciones cardiovasculares dignas de mencionarse son el derrame pericárdico (frecuencia de alrededor de 30\%) aunque rara vez llega al taponamiento cardiaco: de hecho, la mayoría de veces es silente y se resuelve espontáneamente con la realimentación, sin necesidad de intervenciones adicionales (22).

E) Síndrome de realimentación (SR): El bajo peso de las pacientes es un predictor global de la mayor importancia respecto a las complicaciones $(23,24)$. Recuérdese que el IMC $<15$ clasifica a la AN como de extrema gravedad -en el DSM, pero hay consideraciones diferentes en la literatura (25) - y nuestro caso llegó en algún momento a IMC alrededor de 11, y aún menos. La reposición nutricional temprana con micro y macronutrientes y la progresiva recuperación de peso es crucial en reducir la morbimortalidad por AN (14) sin embargo, esta etapa está llena de riesgos a su vez potencialmente letales.

El SR puede definirse como el conjunto de trastornos metabólicos consecuentes a la reiniciación de la alimentación en personas severamente emaciadas. Aunque se describió desde tiempos de Hipócrates, no hay definiciones estandarizadas ni consenso sobre su tratamiento (24). Entre los factores de riesgo para el desarrollo del SR se enumeran IMC $<16$, mínima o ninguna ingesta por más de 10 días, bajos niveles séricos de fósforo, potasio, magnesio, tiamina, antes de reinstalar la dieta, (datos varios que tenía nuestra paciente previamente). Los fenómenos fisiopatológicos subyacentes al SR consisten en 
desequilibrio marcado de electrolitos secundario a la reinstalación de nutrientes y la consiguiente liberación de insulina, pues esta hormona propicia el ingreso masivo de electrolitos como potasio, pero sobre todo el fósforo, a nivel intracelular. El descenso sérico acusado de los niveles de fósforo resulta mortal pues este ión es esencial en los procesos de síntesis de energía (ATP) y la hipofosfatemia conlleva a rabdomiólisis, disfunción eritrocitaria, falla respiratoria y paro cardiaco (14). Literalmente, estaremos ante un colapso cardiopulmonar, no solo por el fracaso de la musculatura respiratoria y miocárdica en producir la energía para su funcionamiento, sino que los volúmenes de dextrosa y solución salina infundidos llegan a desbordar la capacidad del miocardio atrofiado (26) Es probable que en el caso de nuestra paciente, se haya llegado a este extremo y la muerte se haya debido a los efectos del SR en una paciente tan deteriorada por su AN. El SR debe anticiparse y prevenirse en la medida de lo posible pues puede desarrollarse en pocas horas y llevar a un desenlace letal (14).

La muerte súbita en AN, como es comprensible, suele deberse a causa cardiaca. Sin embargo, una serie de diferentes rutas etiopatogénicas pueden confluir en este hecho terminal (26). Incluso la hipoglicemia severa podría presentarse sin los síntomas usuales y llevar a muerte súbita, aunque como factor etiológico por sí sola es rara (27).

En la tabla 1 se muestran algunos criterios selectos respecto a cuándo debiera propiciarse el internamiento de una paciente con AN por el riesgo inminente de su salud ante las complicaciones somáticas descritas. En nuestro país -y en toda Latinoamérica- (25) no existen guías clínicas específicas para el manejo de estas pacientes y debiera sensibilizarse al personal de salud ante la realidad penosa de estos casos que muchas veces acaban en desenlaces letales como el nuestro. Deben hacerse todos los esfuerzos para evitar la repetición de estos desenlaces ominosos: una hospitalización y atención en estadios previos, ya siendo un caso grave $(\mathrm{IMC}<15)$, pero sin esperar a que se acumulen los factores perniciosos como en nuestro caso, podría haber evitado el resultado fatal. La AN es una patología psiquiátrica de implicancia médica que obliga a un conocimiento amplio por parte de todos los implicados en su cuidado. Así lo grafica crudamente Silber cuando recuerda: "Hay consenso acerca de la necesidad de un equipo multidisciplinario para ofrecer la mejor posibilidad de recuperación. La rehabilitación

Tabla 1. Criterios sugerentes de necesidad de internamiento hospitalario para pacientes con AN.

- IMC $<13$ o ingesta $<1000 \mathrm{kcal} /$ día o pérdida de $1 \mathrm{~kg}$ de peso por semana

- Antecedente de síncope

- Frecuencia cardiaca $<40$ x'

- Presión arterial sistólica $<80 \mathrm{~mm} \mathrm{Hg}$

- Presión arterial diastólica $<50 \mathrm{~mm} \mathrm{Hg}$

- Caída postural de PA $>20 \mathrm{~mm} \mathrm{Hg}$

- Intervalo QT corregido $>450 \mathrm{~ms}$

- Temperatura $<35^{\circ} \mathrm{C}$

- Incapacidad de mantenerse en pie

- Vómitos más de 4 veces al día

- Potasio sérico $<2,5 \mathrm{mEq} / \mathrm{L}$

- Sodio sérico $<130 \mathrm{mEq} / \mathrm{L}$

- Glucemia $<60 \mathrm{mg} / \mathrm{dL}$

- Acrocianosis presente

- Elevado riesgo suicida

- Falla de tratamiento ambulatorio o disfunción familiar severa

Modificado de fuentes referenciadas $(25,28)$. 
nutricional es esencial. Declarar que la paciente presenta «progreso psicológico» 0 «comprensión sobre los motivos de su comportamiento» es inaceptable sin una ganancia de peso acompañante. La psicoterapia no es compatible con un cerebro hambriento."

Agradecimiento: A nuestro anónimo revisor o revisora por sus valiosas sugerencias.

Financiamiento: Por los mismos autores.

Conflictos de interés: Los autores declaran no tenerlos.

\section{Correspondencia:}

Lizardo Cruzado

Instituto Nacional de Salud Mental "Honorio Delgado - Hideyo Noguchi”.

Jr. Eloy Espinoza 709. Urb. Palao. San Martín de Porres. Lima 15102. Lima, Perú.

Correo electrónico: lizardo.cruzado.d@upch.pe

Teléfono: (511) 6149205

\section{REFERENCIAS BIBLIOGRÁFICAS}

1. Keel PK, Dorer, DJ, Eddy, KT, Franko DL, Charatan, DL, Herzog DB. Predictors of mortality in eating disorders. Arch Gen Psychiatry. 2003; 60:179-83.

2. Instituto Nacional de Salud Mental. Estudio Epidemiológico de Salud Mental en Niños y Adolescentes en Lima Metropolitana y Callao 2007: Informe General. Anales de Salud Mental. 2012; 28(1):0-0.

3. American Psychiatric Association. Diagnostic and statistical manual of mental disorders. Arlington, VA: American Psychiatric Association; 2013.

4. Keel PK, Forney KJ. Prevalence and incidence of eating disorders in western societies. In: Smolak L, Levine MP (eds.) The Wiley Handbook of Eating Disorders. Chichester: Wiley Blackwell; 2015. pp. 53-63.

5. Steinhausen HC. The outcome of Anorexia Nervosa in the $20^{\text {th }}$ century. Am J Psychiatry. 2002; 159:12841293.

6. Lenoir M, Silber TJ. Anorexia nerviosa en niños y adolescentes (Parte 1). Criterios diagnósticos, historia, epidemiología, etiología, fisiopatología, morbilidad y mortalidad. Arch Argent Pediatr. 2006; 104:253-260.

7. Keel PK. Epidemiology and course of eating disorders. In: Agras WS (ed.) The Oxford Handbook of Eating Disorders. New York: Oxford University Press; 2010. pp. 25-32.

8. Arcelus J, Mitchell AJ, Wales J, Nielsen S. Mortality rates in patients with anorexia nervosa and other eating disorders: A meta-analysis of 36 studies. Arch Gen Psychiatry. 2011;68:724-31.

9. Robinson PH, Nicholls D. Critical care for anorexia nervosa. Cham: Springer; 2015.

10. Castro-Morales J. Anorexia nerviosa en la pobreza. Anales de Salud Mental. 1991; 7:60-74.

11. Gaudiani JL. Medical complications and management strategies in patients with severe restricting and purging. In: Smolak L, Levine MP (eds.) The Wiley Handbook of Eating Disorders. Chichester: Wiley Blackwell; 2015. pp. 712-726.

12. Brown C, Mehler PS. Medical complications of anorexia nervosa and their treatments: an update on some critical aspects. Eat Weight Disord. 2015; 20:419-425.

13. Rosen E, Bakshi N, Walters A, Rosen HR, Mehler PS. Hepatic complications of anorexia nervosa. Dig Dis Sci. 2017; 62: 2977-2981.

14. Hofer M, Pozzi A, Joray M, Ott R, Hähni F, Leuenberger M, et al. Safe refeeding management of anorexia nervosa inpatients: an evidence based protocol. Nutrition. 2014; 30: 524-536.

15. Loung RP, Cooney M, Fallon EM, Langer JC, Katzman DK. Pneumothorax in a young man with anorexia nervosa. Int J Eat Disord. 2016; 49: 895898.

16. Hochlehnert A, Löwe B, Bludau HB, Borst M, Zipfel S, Herzog W. Spontaneous pneumomediastinum in anorexia nervosa: a case report and review of the literature on pneumomediastinum and pneumothorax. Eur Eat Disord Rev. 2010;18:107-15.

17. Albornoz-Muñoz P. Anorexia nerviosa: perfil clínico epidemiológico de pacientes atendidos en el Instituto Nacional de Salud Mental "Honorio Delgado Hideyo Noguchi”, 1982 - 1998. Tesis de Bachiller. Lima, Perú: Universidad Peruana Cayetano Heredia; 1998. $181 \mathrm{pp}$.

18. Harris RH, Sasson G, Mehler PS. Elevation of liver function tests in severe anorexia nervosa. Int $\mathrm{J}$ Eat Disord. 2013; 46: 369-374.

19. Kheloufi M, Boulanger CM, Durand F, Rautou PE. Liver autophagy in anorexia nervosa and acute liver injury. Biomed Res Int. 2014; 2014:701064. doi: 10.1155/2014/701064.

20. Spaulding-Barclay MA, Stern J, Mehler PS. Cardiac changes in anorexia nervosa. Cardiol Young. 2016; 26: 623-628.

21. Di Cola G, Jacoangeli F, Jacoangeli F, Lombardo M, Iellamo F. Cardiovascular disorders in anorexia nervosa and potential therapeutic targets. Intern Emerg Med. 2014; 9: 717-721.

22. Sachs KV, Harnke B, Mehler PS, Krantz MJ. Cardiovascular complications of Anorexia Nervosa: a systematic review. Int J Eat Disord. 2016; 49:238248. 
Anorexia nervosa con desenlace fatal: a propósito de un caso.

23. Treasure J. Applying evidence-based management to anorexia nervosa. Postgrad Med J. 2016; 92:525-531.

24. Wehbe-Chidiac C. An update on the medical consequences of anorexia nervosa. Curr Opin Pediatr. 2019; 31:448-453.

25. Vásquez N, Urrejola P, Vogel M. Actualizaciones en el manejo intrahospitalario de la anorexia nerviosa: recomendaciones prácticas. Rev Med Chile. 2017; 145: 650-656.
26. Mascolo M, Trent S, Colwell C, Mehler PS. What the emergency department needs to know when caring for your patients with eating disorders. Int J Eat Disord. 2012;45:977-81.

27. Jáuregui-Garrido B, Jáuregui-Lobera I. Sudden death in eating disorders. Vasc Health Risk Manag. 2012;8:91-98.

28. Lenoir M, Silber TJ. Anorexia nerviosa en niños y adolescentes (Parte 2). Arch Argent Pediatr. 2006; 104:338-344.

Recibido: 30/11/2019

Aceptado: 02/03/2020 